\title{
硒催化羰基化合成苯氨基甲酸酯和二苯甲烷二氨基甲酸酯
}

\author{
张晓鹏* 荆唤芝彭惟于李亚芳范学森张贵生* \\ (河南师范大学化学化工学院 绿色化学介质与反应省部共建教育部重点实验室 \\ 精细化学品绿色制造河南省协同创新中心 新乡 453007)
}

\begin{abstract}
摘要 首先以廉价易得的非金属硒作催化剂, $\mathrm{CO}$ 替代剧毒光气作羰基化试剂, $\mathrm{O}_{2}$ 作氧化剂, 通过硒催化苯胺与醇 “一 锅煮” 的氧化岸基化反应制得苯氨基甲酸酯; 然后在 $\mathrm{HCl} / \mathrm{ZnCl}_{2}$ 催化下再与甲醛缩合制得二苯甲烷二氨基甲酸酯. 两反 应的目标产物收率从中等到良好, 底物普遍适用性广. $\mathrm{CO}$ 利用率及原子经济性高, 且避免了剧毒光气的使用. 催化剂 硒具有相转移催化作用, 便于回收且能循环使用. 合成方法清洁、经济、高效. 提出了可能的反应机理. 关键词＼cjkstart硒催化; 羰基化; 苯氨基甲酸酯; 二苯甲烷二氨基甲酸酯
\end{abstract}

\section{Selenium-Catalyzed Carbonylation to Phenylcarbamates and Methylene Diphenyl Dicarbamates}

\author{
Zhang, Xiaopeng* \\ Jing, Huanzhi \\ Peng, Weiyu \\ Li, Yafang \\ Fan, Xuesen \\ Zhang, Guisheng* \\ (Collaborative Innovation Center of Henan Province for Green Manufacturing of Fine Chemicals, Key Laboratory of \\ Green Chemical Media and Reactions, Ministry of Education, School of Chemistry and Chemical Engineering,
}

Henan Normal University, Xinxiang 453007)

\begin{abstract}
A clean, economical and efficient approach to phenylcarbamates and methylene diphenyl dicarbamates was reported. With cheap and easily available nonmetal selenium as the catalyst, carbon monoxide instead of virulent phosgene as the carbonylation reagent, oxygen as the oxidant, the selenium-catalyzed oxidative carbonylation reaction of aniline could proceed smoothly with alcohols to afford phenylcarbamates mostly in moderate to good yields. Then, catalyzed by $\mathrm{HCl} / \mathrm{ZnCl} 2$, the condensation of formaldehyde with the generated phenylcarbamates gave methylene diphenyl dicarbamates in moderate to good yields. The applicability of the substrates was good. Catalyst selenium could be easily recovered due to its function of phase-transfer catalysis and could be recycled. High atomic economy, low cost, no emission of corrosive waste, and phosgene-free condition make this approach very promising. The possible reaction mechanisms were also proposed.
\end{abstract}

Keywords selenium-catalysis; carbonylation; phenylcarbamate; methylene diphenyl dicarbamate

苯氨基甲酸酯及二苯甲烷二氨基甲酸酯(MDC)均 属于氨基甲酸酯类化合物，该类化合物在农业上可广泛 用作杀虫剂、除草剂、杀菌剂等 ${ }^{[1]}$; 在化学工业上可替 代剧毒、强腐蚀性的光气法用作清洁制备单苯基异氰酸 酯及二苯甲烷二异氧酸酯的重要前体, 被广泛用于制备 聚氨酯泡沫塑料、人造皮革、胶黏剂、涂料等 ${ }^{[2]}$; 此外, 在 有机合成中该类物质还常用作氨基的保护基 ${ }^{[3]}$.
苯氨基甲酸酯的主要合成方法有: (1)光气法 ${ }^{[4]}$. 醇 先与光气反应生成氯甲酸酯, 然后再与苯胺反应, 或者 苯胺先与光气反应生成苯基异氧酸酯, 然后再与醇加成 制得目标产物，该方法不但用到了剧毒的光气，而且制 备过程中还产生大量的腐蚀性含氯废物, 腐蚀设备及环 境污染严重, 安全性差. (2)光气衍生物及其替代物法 ${ }^{[5]}$. 利用光气衍生物苯基异氰酸酯与醇反应或者氯甲酸酯

* Corresponding author. E-mail: zhangxiaopengv@sina.com; zgs@htu.cn

Received September 12, 2016; revised October 12, 2016; published online October 18, 2016.

Project supported by the Program for Innovative Research Team in Science and Technology in University of Henan Province (No. 15IRTSTHN003), the Young Backbone Teachers Training Fund of Education Department of Henan Province (No. 2013GGJS-059) and the Young Backbone Teachers Training Fund of Henan Normal University (No. 2011-8).

河南省高校科技创新团队支持计划(No. 15IRTSTHN003)、河南省高等学校青年骨干教师(No. 2013GGJS-059)和河南师范大学青年骨干教师(No. 2011-8)资助项目. 
与苯胺反应可制得目标产物, 或者用三光气、羰基二咪 唑或碳酸酯等替代光气来合成目标产物, 但该类方法中 作为反应起始原料的异氧酸酯及氯甲酸酯为光气衍生 物, 相当于间接使用了光气, 而其余光气替代物则通常 是其中的崖基进入到了目标产物中, 其余部分大多变成 了副产物, 故反应的原子经济性低, 成本高. (3)还原羰 基化法 ${ }^{[2 a, 2 b, 6]}$. 硝基苯与醇在 $\mathrm{CO}$ 存在下通过催化还原羰 基化反应得到目标产物, 副产物为 $\mathrm{CO}_{2}$. 该方法用 $\mathrm{CO}$ 替代光气及其衍生物, 具有宸基化试剂来源方便、毒性 相对较低、无腐蚀性废物排放、原子经济性较高等诸多 优点, 但该方法所用的催化剂通常为钯、钉、铑等的复 杂配合物, 价格高且难以重复使用; 廉价易得的非金属 硒也被用于催化该反应 ${ }^{[7]}$, 反应效果良好, 但该还原羰 基化法的另一缺陷是反应消耗的 $\mathrm{CO}$ 只有三分之一转化 到了产物中去, 其余参与反应的 $\mathrm{CO}$ 则转变成了温室气 体 $\mathrm{CO}_{2}$, 导致 $\mathrm{CO}$ 的有效利用率低, 而且要将反应体系 中多余的 $\mathrm{CO}$ 与生成的 $\mathrm{CO}_{2}$ 分离回收则相对困难, 会增 加反应的操作成本. (4)氧化羰基化法 ${ }^{[8]}$. 该方法通常以 苯胺和醇为起始原料, 在 $\mathrm{CO}$ 和 $\mathrm{O}_{2}$ 存在下经催化氧化羰 基化反应制得目标产物, 副产物为清洁无污染的水. 该 方法 $\mathrm{CO}$ 利用率高, 无腐蚀性废物或 $\mathrm{CO}_{2}$ 生成, 多余的 CO 回收方便, 但该方法所用的催化剂通常为钯、钓、 钛、金等的盐或其配合物, 同样存在价格高且难以重复 使用等缺陷.二苯甲烷二氨基甲酸酯的主要合成方法总 体上有二: (1)由二苯甲烷二胺或其光气衍生物二苯甲烷 二异氰酸酯经类似上述苯氨基甲酸酯的合成方法制

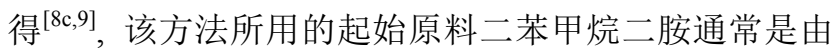
苯胺与甲醛缩合制得. (2)经前述方法制得的苯氨基甲酸 酯再与甲醛缩合制得 ${ }^{[10]}$. 由此可见, 本文所涉及的苯氨 基甲酸酯及二苯甲烷二氨基甲酸酯的核心内容均为氨 基甲酸酯的制备. 而目前该类化合物的制备方法中, 要 么直接或间接使用了剧毒、强腐蚀性的光气, 要么存在 催化剂昂贵且难以重复利用、原子经济性低、成本高、 $\mathrm{CO}$ 利用率低等缺陷, 因此积极研究开发一条非光气、 催化剂廉价易得、原子经济性高、成本低、 $\mathrm{CO}$ 利用率 高的氨基甲酸酯的清洁、经济、高效合成法将具有重要 意义.

研究发现 ${ }^{[11]}$, 非金属硒可有效活化 CO 来替代光气 及其衍生物进行羰基化反应, 具有毒性相对较低、无腐 蚀性废物排放、原子经济性高、环境相对友好等优点; 与 贵金属催化羰基化法相比, 硒廉价易得且具有相转移催 化功能, 分离回收方便, 回收的硒可循环使用, 其催化 活性保持不变, 因而能显著降低反应成本. 鉴于此, 本 文拟将 $\mathrm{Se} / \mathrm{CO}$ 催化体系引入到苯氨基甲酸酯及二苯甲 烷二氨基甲酸酯的合成中来, 以市售的苯胺和醇为起始
原料，在 $\mathrm{O}_{2}$ 存在下经硒催化 “一锅煮” 的氧化羰基化反 应来合成苯氨基甲酸酯, 生成的苯氨基甲酸酯再与甲醛 缩合制得二苯甲烷二氨基甲酸酯(Scheme 1), 以期为这 两类氨基甲酸酯的合成开辟出一条清洁、经济、高效的 新途径.

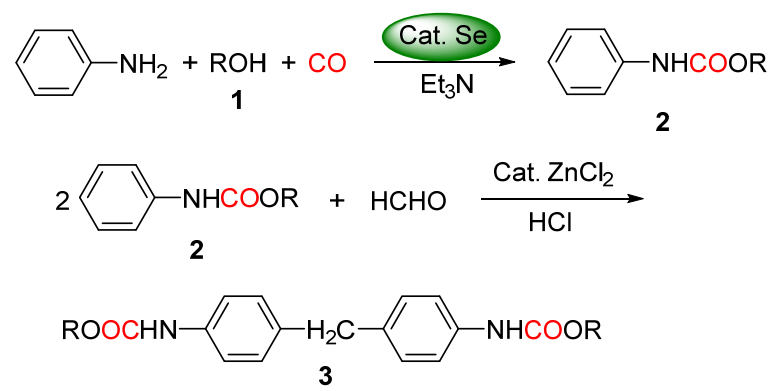

图式 1 硒催化羰基化合成苯氨基甲酸酯和二苯甲烷二氨基 甲酸酯

Scheme 1 Selenium-catalyzed carbonylation to phenylcarbamates and methylene diphenyl dicarbamates

\section{1 结果与讨论}

\section{1 合成苯氨基甲酸酯反应的底物适用性}

为了探寻硒催化氧化羰基化合成苯氨基甲酸酯反 应的底物适用性, 本文尝试用一系列醇作底物与苯胺进 行硒催化的氧化羰基化反应，结果如表 1 所示.

实验发现, 大多数醇均可与苯胺顺利发生硒催化的 氧化羰基化反应，能以中等到良好的分离收率得到目标 产物苯氨基甲酸酯. 空间效应对反应影响显著：当空间 位阻小的醇如短碳链的正构醇乙醇、丙醇、丁醇、戊醇 等用于该催化体系应时，反应能很好地进行，可以良好 收率得到目标产物(表 1, Entries 1, 2, 4, 7). 随着碳链的 增长, 醇的反应活性逐渐降低, 导致目标产物的收率也 相应地降低(表 1, Entries 10,12,13), 这很可能是由于随 着碳链的增长, 碳链在反应体系中的扭曲程度也相应增 加从而导致其空间位阻增大所致.同理，空间效应也导 致正构醇比其支链异构体或环状异构体的反应活性要 高(表 1, Entries 2 vs 3; 4 vs 5 and $6 ; 7$ vs 8 and 9; 10 vs 11 ), 叔丁醇、叔戊醇由于其空间位阻太大难以进行该催 化羰基化反应，因而未能得到相应的目标产物(表 1 , Entries 6, 9).

\section{2 合成二苯甲烷二氨基甲酸酯反应的底物适用性}

用盐酸和氯化锌作为催化剂, 将上述合成的一系列 苯氨基甲酸酯作起始原料, 分别在相应醇中与甲醛缩合 制备二苯甲烷二氨基甲酸酯, 结果如表 2 所示.

反应结果表明, 在盐酸和氯化锌催化下, 上述合成 的一系列苯氨基甲酸酯在相应醇中可顺利实现与甲醛 的缩合反应，均能以中等到良好的分离收率得到目标产 
表 1 合成苯氨基甲酸酯反应的底物范围 ${ }^{a}$

Table 1 Substrates scope for preparation of phenylcarbamates

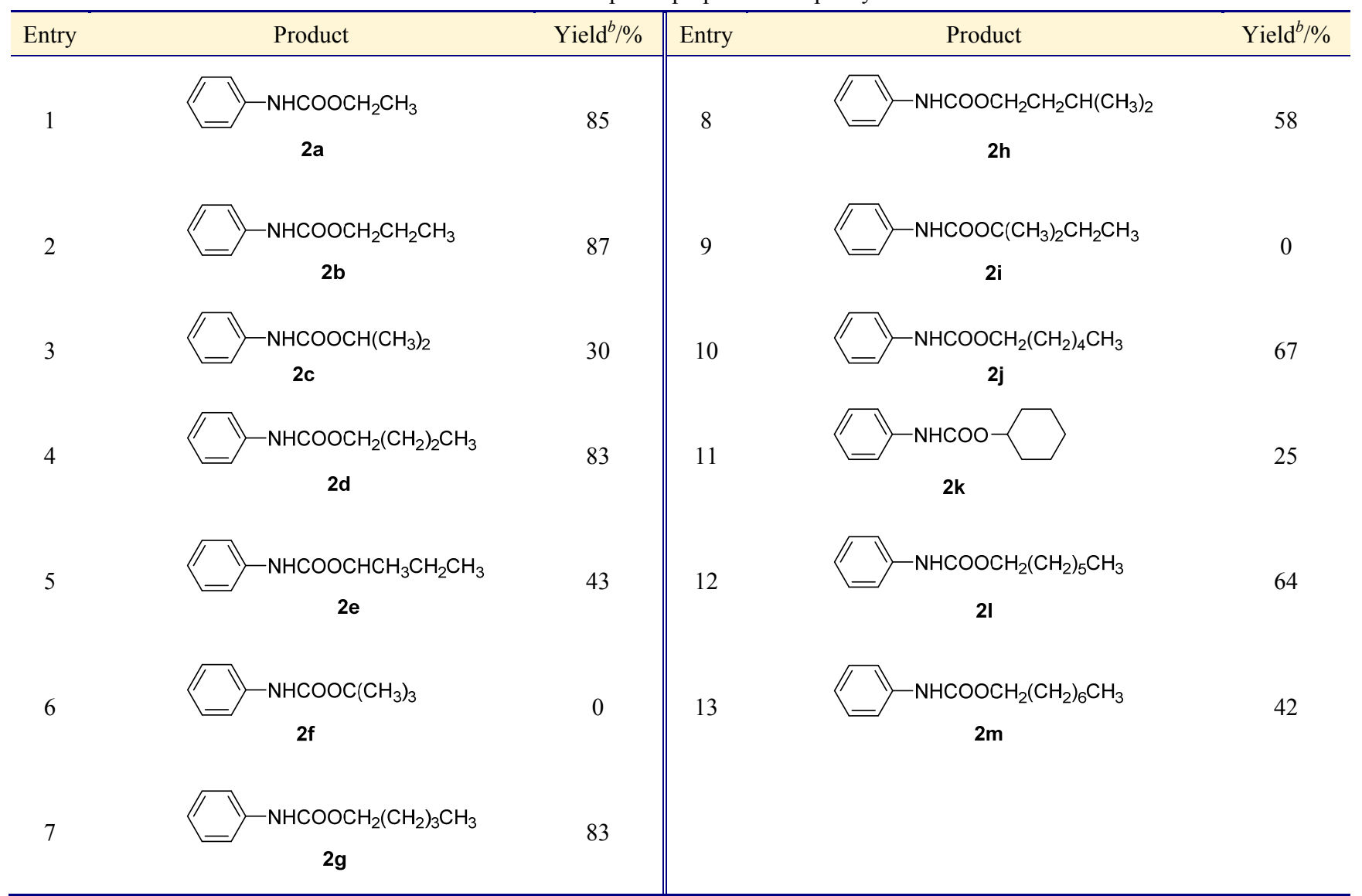

${ }^{a}$ All the reactions were carried out with aniline $(5 \mathrm{mmol})$, alcohol $(50 \mathrm{mmol}), \mathrm{Se}(0.25 \mathrm{mmol}), \mathrm{Et}_{3} \mathrm{~N}(10 \mathrm{mmol})$, mixed gas of CO and $\mathrm{O}_{2}\left[2 \mathrm{MPa}, n(\mathrm{CO}): n\left(\mathrm{O}_{2}\right)=\right.$ $4: 1]$ at $180{ }^{\circ} \mathrm{C}$ for $6 \mathrm{~h} ;{ }^{b}$ Isolated yield.

物二苯甲烷二氨基甲酸酯(表 2 , Entries $1 \sim 11$ ), 底物的 普遍适用性广泛、良好. 尤其是由短链正构醇参与形成 的苯氨基甲酸酯在和甲醛进行缩合反应时活性较高, 目 标产物收率良好(表 2, Entries 1, 2, 4, 7). 醇在此反应体 系中的作用有二: 一是可用作反应的溶剂, 二是在反应 过程中也可抑制苯氨基甲酸酯受热可能分解成异氰酸 酯和醇的副反应.

\section{3 反应机理}

如 Scheme 2 所示, 在 $\mathrm{Et}_{3} \mathrm{~N}$ 存在下 $\mathrm{Se}$ 首先与 $\mathrm{CO}$ 反 应原位生成 $\mathrm{SeCO}(\mathbf{I})^{[11]}$ 从而引发随后的羰基化反应, I 生成后分别接受苯胺和醇的亲核进攻生成中间体 II, 随 后 II 经消除反应生成苯氨基甲酸酯(III), 同时释放出 $\mathrm{H}_{2} \mathrm{Se}(\mathbf{I V})$, IV 随后被 $\mathrm{O}_{2}$ 氧化分解为 $\mathrm{Se}$ 和 $\mathrm{H}_{2} \mathrm{O}$ 参与下一 轮的催化循环反应 ${ }^{[12]}$; 甲醛在催化剂 $\mathrm{HCl}$ 及 $\mathrm{ZnCl}_{2}$ 作用 下首先形成亲电性更强的物种 $\mathbf{V}$, 随后 $\mathbf{V}$ 亲电进攻 III 的苯环生成 4-差㙁甲基苯氨基甲酸酯(VI), VI 生成后其羟 甲基中的羟基在酸作用下被质子化, 进而脱去 $\mathrm{H}_{2} \mathrm{O}$ 形成 亲电物种 VII, VII 再对另一分子的 III 进行亲电加成, 脱去质子即得最终产物二苯甲烷二氨基甲酸酯(MDC,
VIII ${ }^{[13]}$.

\section{2 结论}

成功开发出一种清洁、经济、高效的合成苯氨基甲 酸酯及二苯甲烷二氨基甲酸酯的新方法. 以非金属硒作 催化剂, $\mathrm{CO}$ 作羰基化试剂, $\mathrm{O}_{2}$ 作氧化剂, 硒催化苯胺与 醇特别是空间位阻小的醇的氧化羰基化反应可顺利进 行, 能以中等到良好的收率得到苯氨基甲酸酯; 在 $\mathrm{HCl} / \mathrm{ZnCl}_{2}$ 催化下苯氨基甲酸酯可顺利与甲醛发生缩合 反应制得二苯甲烷二氨基甲酸酯, 收率从中等到良好. 催化剂硒廉价易得又便于回收循环使用，其催化活性保 持不变; 用 $\mathrm{CO}$ 替代剧毒、强腐蚀性的光气，且其利用 率高, 无腐蚀性废物或温室气体 $\mathrm{CO}_{2}$ 排放, 环境相对友 好，原子经济性高，成本低; 反应路线简洁，操作方便; 两反应底物的普遍适用性良好.

\section{3 实验部分}

\section{1 仪器与试剂}

CO 钢瓶气体(纯度为 $99.9 \%$, 北京普莱克斯实用气 
表 2 合成二苯甲烷二氨基甲酸酯反应的底物范围 ${ }^{a}$

Table 2 Substrates scope for preapreation of methylene diphenyl dicarbamates

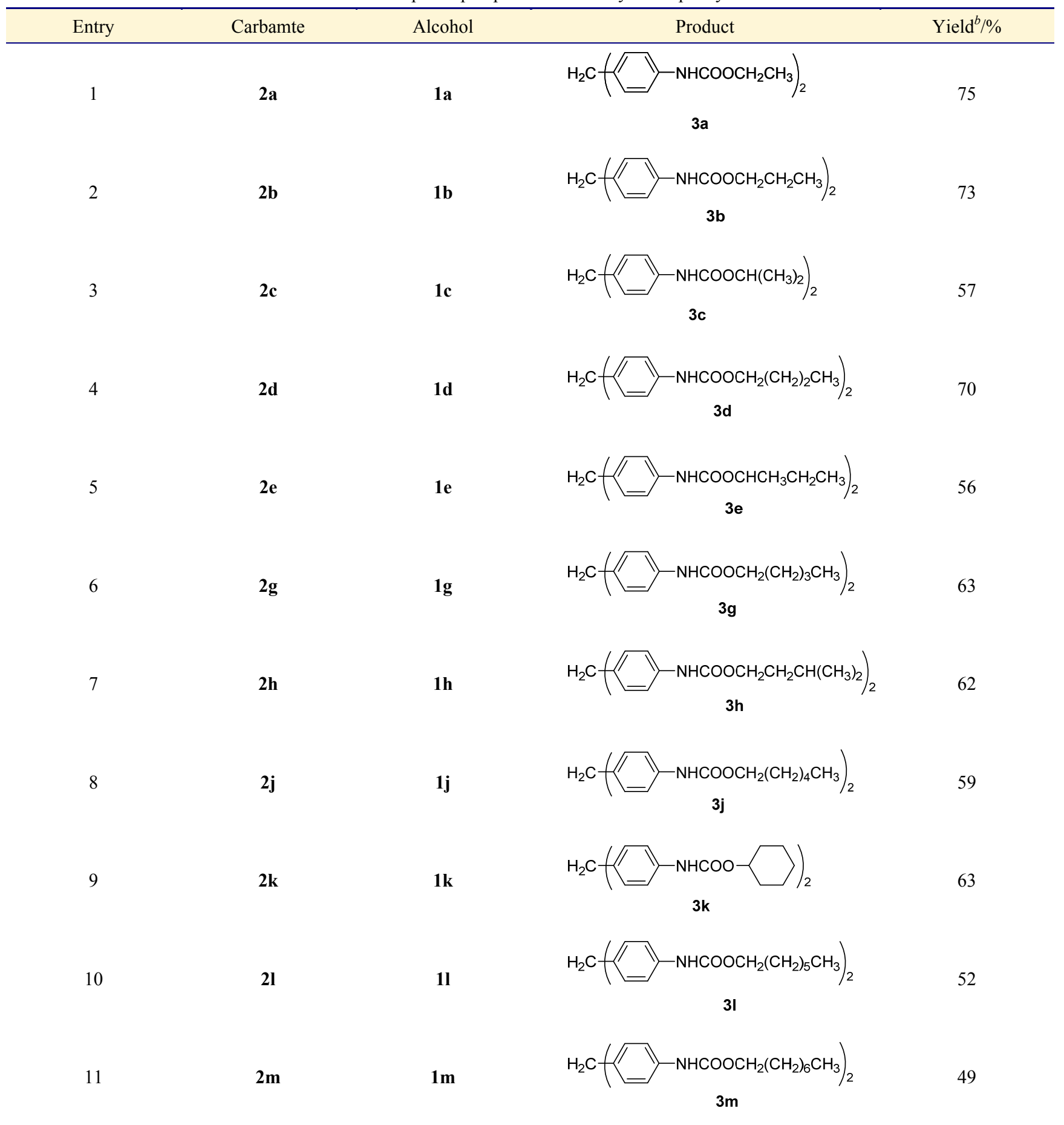

${ }^{a}$ All the reactions were carried out with phenylcarbamate $(5 \mathrm{mmol}), \mathrm{HCHO}(20 \mathrm{mmol})$, alcohol $(25 \mathrm{mmol}), \mathrm{ZnCl}_{2}(2 \mathrm{mmol}), \mathrm{HCl}(36 \%, 3 \mathrm{~mL})$ at $80{ }^{\circ} \mathrm{C}$ for $6 \mathrm{~h}$; ${ }^{b}$ Isolated yield.

体有限公司), $\mathrm{O}_{2}$ 钢瓶气体(纯度为 $99.9 \%$, 新乡北普特种 气体有限公司), 硒粉(纯度为 $99.95 \%$, 上海兴塔化工 厂); 其余药品及试剂均为市售分析纯, 使用前未做进 一步处理.

XT4 数字显示显微熔点仪(北京科仪电光仪器厂, 温度未经校正); Bruker DPX-400 核磁共振谱仪(内标
TMS，溶剂 $\mathrm{CDCl}_{3}$ 或 DMSO- $d_{6}$, 北京百灵威科技有限公 司).

\section{2 苯氨基甲酸酯的合成}

将硒粉 $(0.25 \mathrm{mmol}) 、$ 苯胺 $(5 \mathrm{mmol}) 、$ 醇 $(1,50 \mathrm{mmol})$ 、 三乙胺 $(10 \mathrm{mmol})$ 依次加入 $100 \mathrm{~mL}$ 高压反应釜中, 密封, 用 $\mathrm{CO}$ 与 $\mathrm{O}_{2}$ 的混合气体 $\left[n(\mathrm{CO}): n\left(\mathrm{O}_{2}\right)=4: 1\right]$ 置换其内 


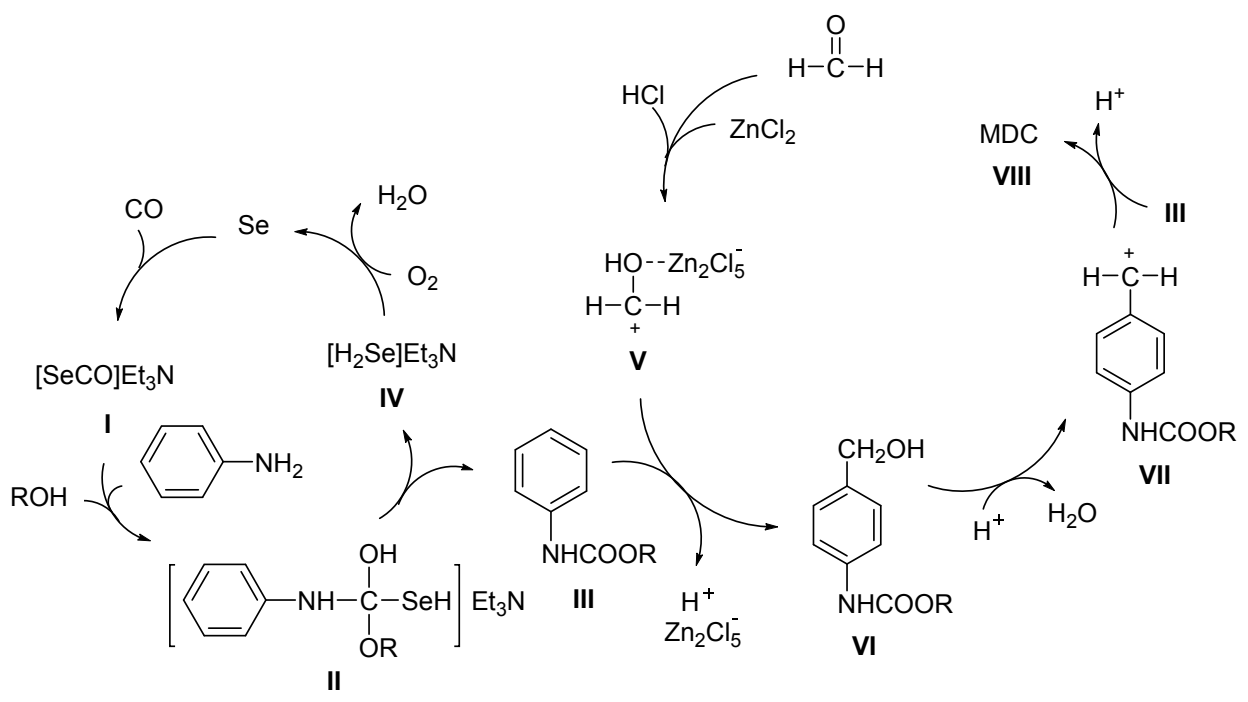

图式 2 生成苯氨基甲酸酯和二苯甲烷二氨基甲酸酯的可能机理

Scheme 2 Proposed reaction pathway to phenylcarbamates and methylene diphenyl dicarbamates

空气 3 次后, 再将此混合气体充入反应釜至 $2 \mathrm{MPa}$. 随 后将此反应釜放入 $180{ }^{\circ} \mathrm{C}$ 的恒温油浴中, 开启磁力摚 拌器进行反应 $6 \mathrm{~h}$. 反应完成后停止加热, 并将反应釜 冷却至室温, 然后放掉残余气体, 开釜, 将釜内反应液 置于空气中再摚拌一段时间以使硒充分沉淀析出, 然后 经抽滤回收单质硒. 滤液浓缩后经柱色谱分离[淋洗液: $V($ 石油醚 $) ： V($ 乙酸乙酯 $)=10 \sim 15 ： 1$ ] 即可得到目标产 物苯氨基甲酸酯(2).

苯氨基甲酸乙酯(2a): 白色针晶. m.p. $49 \sim 50{ }^{\circ} \mathrm{C}$ (lit. ${ }^{[7]} 51 \sim 53{ }^{\circ} \mathrm{C}$ ); ${ }^{1} \mathrm{H}$ NMR (DMSO- $\left.d_{6}, 400 \mathrm{MHz}\right) \delta: 9.61$ (brs, $1 \mathrm{H}), 7.46 \sim 6.96(\mathrm{~m}, 5 \mathrm{H}), 4.12(\mathrm{q}, J=7.2 \mathrm{~Hz}, 2 \mathrm{H})$, $1.24(\mathrm{t}, J=7.2 \mathrm{~Hz}, 3 \mathrm{H})$.

苯氨基甲酸丙酯 $(\mathbf{2 b})$ : 白色针晶. m.p. $51 \sim 52{ }^{\circ} \mathrm{C}$ (lit. $\left.{ }^{[7]} 51 \sim 53{ }^{\circ} \mathrm{C}\right) ;{ }^{1} \mathrm{H}$ NMR $\left(\mathrm{CDCl}_{3}, 400 \mathrm{MHz}\right) \delta: 6.63$ (brs, $1 \mathrm{H}), 7.40 \sim 7.05(\mathrm{~m}, 5 \mathrm{H}), 4.14(\mathrm{t}, J=6.8 \mathrm{~Hz}, 2 \mathrm{H})$, $1.74 \sim 1.68(\mathrm{~m}, 2 \mathrm{H}), 0.99(\mathrm{t}, J=7.4 \mathrm{~Hz}, 3 \mathrm{H})$.

苯氨基甲酸异丙酯 $(\mathbf{2 c})$ : 白色针晶. m.p. $82{ }^{\circ} \mathrm{C}$ (lit. $\left.{ }^{[14]} 82 \sim 83{ }^{\circ} \mathrm{C}\right) ;{ }^{1} \mathrm{H} \mathrm{NMR}\left(\mathrm{CDCl}_{3}, 400 \mathrm{MHz}\right) \delta: 6.53$ (brs, $1 \mathrm{H}), 7.39 \sim 7.04(\mathrm{~m}, 5 \mathrm{H}), 5.06 \sim 4.99(\mathrm{~m}, 1 \mathrm{H}), 1.31$ (d, $J=6.0 \mathrm{~Hz}, 6 \mathrm{H})$.

苯氨基甲酸丁酯(2d): 白色针晶. m.p. $61 \sim 62{ }^{\circ} \mathrm{C}$ (lit. $\left.{ }^{[7]} 65{ }^{\circ} \mathrm{C}\right) ;{ }^{1} \mathrm{H}$ NMR $\left(\mathrm{CDCl}_{3}, 400 \mathrm{MHz}\right) \delta: 6.76$ (brs, 1H), $7.42 \sim 7.06(\mathrm{~m}, 5 \mathrm{H}), 4.14(\mathrm{t}, J=6.8 \mathrm{~Hz}, 2 \mathrm{H}), 1.71 \sim$ $1.64(\mathrm{~m}, 2 \mathrm{H}), 1.49 \sim 1.39(\mathrm{~m}, 2 \mathrm{H}), 0.98(\mathrm{t}, J=7.4 \mathrm{~Hz}, 3 \mathrm{H})$.

苯氨基甲酸仲丁酯 (2e): 白色针晶. m.p. $65{ }^{\circ} \mathrm{C}$ (lit. $\left.{ }^{[15]} 66 \sim 67{ }^{\circ} \mathrm{C}\right) ;{ }^{1} \mathrm{H} \mathrm{NMR}\left(\mathrm{CDCl}_{3}, 400 \mathrm{MHz}\right) \delta: 6.60$ (brs, $1 \mathrm{H}), 7.42 \sim 7.05(\mathrm{~m}, 5 \mathrm{H}), 4.89 \sim 4.85(\mathrm{~m}, 1 \mathrm{H}), 1.71 \sim$ $1.56(\mathrm{~m}, 2 \mathrm{H}), 1.29$ (d, $J=6.0 \mathrm{~Hz}, 3 \mathrm{H}), 0.97$ (t, $J=7.6 \mathrm{~Hz}$, $3 \mathrm{H})$. 苯氨基甲酸戊酯(2g): 白色针晶. m.p. $47{ }^{\circ} \mathrm{C}$ (lit. ${ }^{[16]}$ $\left.48{ }^{\circ} \mathrm{C}\right) ;{ }^{1} \mathrm{H}$ NMR $\left(\mathrm{CDCl}_{3}, 400 \mathrm{MHz}\right) \delta: 6.62$ (brs, $1 \mathrm{H}$ ), $7.41 \sim 7.06(\mathrm{~m}, 5 \mathrm{H}), 4.18(\mathrm{t}, J=6.8 \mathrm{~Hz}, 2 \mathrm{H}), 1.73 \sim 1.66$ (m, 2H), $1.42 \sim 1.34(\mathrm{~m}, 4 \mathrm{H}), 0.94(\mathrm{t}, J=5.6 \mathrm{~Hz}, 3 \mathrm{H})$.

苯氨基甲酸异戊酯 (2h): 白色针晶. m.p. $51{ }^{\circ} \mathrm{C}$ (lit. $\left.{ }^{[17]} 55{ }^{\circ} \mathrm{C}\right) ;{ }^{1} \mathrm{H}$ NMR $\left(\mathrm{CDCl}_{3}, 400 \mathrm{MHz}\right) \delta: 7.18$ (brs, $1 \mathrm{H}), 7.45 \sim 7.06(\mathrm{~m}, 5 \mathrm{H}), 4.24(\mathrm{t}, J=6.8 \mathrm{~Hz}, 2 \mathrm{H}), 1.78 \sim$ $1.72(\mathrm{~m}, 1 \mathrm{H}), 1.62 \sim 1.56(\mathrm{~m}, 2 \mathrm{H}), 0.99(\mathrm{t}, J=4.8 \mathrm{~Hz}, 6 \mathrm{H})$.

苯氨基甲酸己酯 $(\mathbf{2 j})$ : 白色针晶. m.p. $42{ }^{\circ} \mathrm{C}$ (lit. ${ }^{[7]}$ $44 \sim 45{ }^{\circ} \mathrm{C}$ ); ${ }^{1} \mathrm{H}$ NMR (DMSO- $\left.d_{6}, 400 \mathrm{MHz}\right) \delta$ : 9.56 (brs, $1 \mathrm{H}), 7.47 \sim 6.95(\mathrm{~m}, 5 \mathrm{H}), 4.06(\mathrm{t}, J=6.6 \mathrm{~Hz}, 2 \mathrm{H}), 1.64 \sim$ $1.57(\mathrm{~m}, 2 \mathrm{H}), 1.39 \sim 1.27(\mathrm{~m}, 6 \mathrm{H}), 0.88(\mathrm{t}, J=6.8 \mathrm{~Hz}, 3 \mathrm{H})$.

苯氨基甲酸环己酯(2k): 白色针晶. m.p. $80 \sim 81{ }^{\circ} \mathrm{C}$ (lit. ${ }^{[7]} 81 \sim 83{ }^{\circ} \mathrm{C}$ ); ${ }^{1} \mathrm{H}$ NMR (DMSO- $\left.d_{6}, 400 \mathrm{MHz}\right) \delta$ : 9.55 (brs, $1 \mathrm{H}), 7.47 \sim 6.94(\mathrm{~m}, 5 \mathrm{H}), 4.59 \sim 4.64(\mathrm{~m}, 1 \mathrm{H}), 1.90 \sim$ $1.21(\mathrm{~m}, 10 \mathrm{H})$.

苯氨基甲酸庚酯(2I): 白色针晶. m.p. $57{ }^{\circ} \mathrm{C}$ (lit. ${ }^{[18]}$ $\left.58 \sim 58.5{ }^{\circ} \mathrm{C}\right) ;{ }^{1} \mathrm{H}$ NMR $\left(\mathrm{CDCl}_{3}, 400 \mathrm{MHz}\right) \delta: 6.60$ (brs, $1 \mathrm{H}), 7.40 \sim 7.05(\mathrm{~m}, 5 \mathrm{H}), 4.17(\mathrm{t}, J=6.8 \mathrm{~Hz}, 2 \mathrm{H}), 1.70 \sim$ $1.60(\mathrm{~m}, 2 \mathrm{H}), 1.41 \sim 1.31(\mathrm{~m}, 8 \mathrm{H}), 0.90(\mathrm{t}, J=6.8 \mathrm{~Hz}, 3 \mathrm{H})$.

苯氨基甲酸辛酯 $(\mathbf{2 m})$ : 白色针晶. m.p. $72{ }^{\circ} \mathrm{C}$ (lit. ${ }^{[19]}$ 72 74 $\left.{ }^{\circ} \mathrm{C}\right) ;{ }^{1} \mathrm{H}$ NMR $\left(\mathrm{CDCl}_{3}, 400 \mathrm{MHz}\right) \delta: 6.59$ (brs, $1 \mathrm{H}), 7.40 \sim 7.05(\mathrm{~m}, 5 \mathrm{H}), 4.17(\mathrm{t}, J=7.0 \mathrm{~Hz}, 2 \mathrm{H}), 1.71 \sim$ $1.64(\mathrm{~m}, 2 \mathrm{H}), 1.39 \sim 1.29(\mathrm{~m}, 10 \mathrm{H}), 0.89(\mathrm{t}, J=6.4 \mathrm{~Hz}$, $3 \mathrm{H})$.

\section{3 二苯甲烷二氨基甲酸酯的合成}

将苯氨基甲酸酯 $(5 \mathrm{mmol}) 、$ 醇 $(25 \mathrm{mmol}) 、$ 盐酸 $36 \%$, $3 \mathrm{~mL}) 、$ 氯化锌 $(2 \mathrm{mmol})$ 依次加入装有冷凝管的 $100 \mathrm{~mL}$ 三颈烧瓶中, 然后开启磁力搅拌器, 当温度上升至 
$45{ }^{\circ} \mathrm{C}$ 时, 将甲醛( $37 \%, 20 \mathrm{mmol}$ )逐滴加到反应器内, 滴 加过程中确保温度不变. 滴加完毕后, 将温度迅速升到 $80{ }^{\circ} \mathrm{C}$ 并维持此温度反应 $6 \mathrm{~h}$. 反应结束后将反应液冷却 至室温, 先用氢氧化钠溶液中和, 再用三氯甲烷萃取, 然后经干燥、过滤、旋蒸除去三氯甲烷后经柱色谱分离 [淋洗液: $V$ (石油醚) $: V$ (乙酸乙酯 $)=15 ： 1$ ] 即可得到二 苯甲烷二氨基甲酸酯(3).

二苯甲烷二氨基甲酸二乙酯(3a): 白色固体. m.p. $129 \sim 130{ }^{\circ} \mathrm{C}$ (lit. $\left.{ }^{[20]} 133 \sim 134{ }^{\circ} \mathrm{C}\right)$; ${ }^{1} \mathrm{H}$ NMR $\left(\mathrm{CDCl}_{3}\right.$, $400 \mathrm{MHz}) \delta: 9.61$ (brs, $2 \mathrm{H}), 7.46 \sim 6.96(\mathrm{~m}, 8 \mathrm{H}), 4.21$ (q, $J=7.2 \mathrm{~Hz}, 4 \mathrm{H}), 3.88(\mathrm{~s}, 2 \mathrm{H}), 1.28(\mathrm{t}, J=7.2 \mathrm{~Hz}, 6 \mathrm{H}) ;{ }^{13} \mathrm{C}$ $\mathrm{NMR}\left(\mathrm{CDCl}_{3}, 100 \mathrm{MHz}\right) \delta: 153.66,136.26,136.00$, $129.41,118.91,61.17,40.54,14.56$.

二苯甲烷二氨基甲酸二丙酯 $(3 b)$ : 白色固体. m.p. 99 $100{ }^{\circ} \mathrm{C}$ (lit. $\left.{ }^{[22]} 150 \sim 152{ }^{\circ} \mathrm{C}\right)$; ${ }^{1} \mathrm{H}$ NMR $\left(\mathrm{CDCl}_{3}, 400\right.$ MHz) $\delta: 7.30 \sim 7.06(\mathrm{~m}, 8 \mathrm{H}), 6.58$ (brs, 2H), $4.09(\mathrm{t}, J=$ $6.8 \mathrm{~Hz}, 4 \mathrm{H}), 3.88(\mathrm{~s}, 2 \mathrm{H}), 1.73 \sim 1.64(\mathrm{~m}, 4 \mathrm{H}), 0.97(\mathrm{t}, J=$ $7.4 \mathrm{~Hz}, 6 \mathrm{H})$.

二苯甲烷二氨基甲酸二异丙酯 $(3 \mathbf{c})$ : 白色固体. m.p. $137 \sim 138{ }^{\circ} \mathrm{C} \quad\left(\right.$ lit. $\left.^{[22]} 150 \sim 152{ }^{\circ} \mathrm{C}\right) ;{ }^{1} \mathrm{H}$ NMR $\left(\mathrm{CDCl}_{3}\right.$, $400 \mathrm{MHz}) \delta: 7.30 \sim 7.09(\mathrm{~m}, 8 \mathrm{H}), 6.50$ (brs, 2H), 5.05 $5.00(\mathrm{~m}, 2 \mathrm{H}), 3.89$ (s, 2H), 1.27 (d, J=7.8 Hz, 12H).

二苯甲烷二氨基甲酸二丁酯(3d): 白色固体. m.p. $100 \sim 102{ }^{\circ} \mathrm{C}$ (lit. $\left.{ }^{[23]} 112{ }^{\circ} \mathrm{C}\right) ;{ }^{1} \mathrm{H}$ NMR $\left(\mathrm{CDCl}_{3}, 400\right.$ $\mathrm{MHz}) \delta: 7.29 \sim 7.06(\mathrm{~m}, 8 \mathrm{H}), 6.57$ (brs, $2 \mathrm{H}), 4.15(\mathrm{t}, \quad J=$ $6.4 \mathrm{~Hz}, 4 \mathrm{H},), 3.88(\mathrm{~s}, 2 \mathrm{H}), 1.68 \sim 1.59(\mathrm{~m}, 4 \mathrm{H}), 1.45 \sim 1.35$ (m, 4H), $0.95(\mathrm{t}, J=7.2 \mathrm{~Hz}, 6 \mathrm{H})$.

二苯甲烷二氨基甲酸二仲丁酯(3e): 白色固体. m.p. $141 \sim 142{ }^{\circ} \mathrm{C} \quad\left(\mathrm{CAS}\right.$ No. 1246888-42-5); ${ }^{1} \mathrm{H} \quad \mathrm{NMR}$ $\left(\mathrm{CDCl}_{3}, 400 \mathrm{MHz}\right) \delta: 7.31 \sim 7.09(\mathrm{~m}, 8 \mathrm{H}), 6.55$ (brs, $\left.2 \mathrm{H}\right)$, $4.87 \sim 4.82(\mathrm{~m}, 2 \mathrm{H}), 3.89(\mathrm{~s}, 2 \mathrm{H}), 1.67 \sim 1.57(\mathrm{~m}, 4 \mathrm{H})$, $1.27(\mathrm{~d}, J=6.4 \mathrm{~Hz}, 6 \mathrm{H}), 0.94(\mathrm{t}, J=7.4 \mathrm{~Hz}, 6 \mathrm{H})$.

二苯甲烷二氨基甲酸二戊酯 $(\mathbf{3 g})$ : 白色固体. m.p. $114{ }^{\circ} \mathrm{C}$ (CAS No. 102894-35-9); ${ }^{1} \mathrm{H}$ NMR $\left(\mathrm{CDCl}_{3}, 400\right.$ $\mathrm{MHz}) \delta: 7.30 \sim 7.10(\mathrm{~m}, 8 \mathrm{H}), 6.52(\mathrm{brs}, 2 \mathrm{H}), 4.15(\mathrm{t}, J=$ $6.8 \mathrm{~Hz}, 4 \mathrm{H}), 3.89(\mathrm{~s}, 2 \mathrm{H}), 1.69 \sim 1.65(\mathrm{~m}, 4 \mathrm{H}), 1.39 \sim 1.35$ $(\mathrm{m}, 8 \mathrm{H}), 0.92(\mathrm{t}, J=6.8 \mathrm{~Hz}, 6 \mathrm{H})$.

二苯甲烷二氨基甲酸二异戊酯(3h): 白色固体. m.p. $91{ }^{\circ} \mathrm{C}$ (CAS No. 102894-34-8); ${ }^{1} \mathrm{H}$ NMR $\left(\mathrm{CDCl}_{3}, 400\right.$ $\mathrm{MHz}) \delta: 7.30 \sim 7.09(\mathrm{~m}, 8 \mathrm{H}), 6.59$ (brs, $2 \mathrm{H}), 4.19(\mathrm{t}, J=$ $7.0 \mathrm{~Hz}, 4 \mathrm{H}), 3.89(\mathrm{~s}, 2 \mathrm{H}), 1.73 \sim 1.71(\mathrm{~m}, 2 \mathrm{H}), 1.59 \sim 1.55$ $(\mathrm{m}, 4 \mathrm{H}), 0.94(\mathrm{t}, d=6.4 \mathrm{~Hz}, 12 \mathrm{H})$.

二苯甲烷二氨基甲酸二己酯 $(\mathbf{3} \mathbf{j})$ : 白色固体. m.p. $129{ }^{\circ} \mathrm{C}$ (lit. $\left.{ }^{[22]} 108 \sim 110{ }^{\circ} \mathrm{C}\right) ;{ }^{1} \mathrm{H}$ NMR $\left(\mathrm{CDCl}_{3}, 400\right.$ MHz) $\delta: 7.30 \sim 7.09(\mathrm{~m}, 8 \mathrm{H}), 6.57$ (brs, $2 \mathrm{H}), 4.15(\mathrm{t}, J=$
$6.8 \mathrm{~Hz}, 4 \mathrm{H}), 3.89(\mathrm{~s}, 2 \mathrm{H}), 1.70 \sim 1.63(\mathrm{~m}, 4 \mathrm{H}), 1.42 \sim 1.23$ $(\mathrm{m}, 12 \mathrm{H}), 0.90(\mathrm{t}, J=6.8 \mathrm{~Hz}, 6 \mathrm{H})$.

二苯甲烷二氨基甲酸二环己酯(3k): 白色固体. m.p. 90 91 ${ }^{\circ} \mathrm{C}$ (CAS No. 76788-43-7); ${ }^{1} \mathrm{H}$ NMR $\left(\mathrm{CDCl}_{3}, 400\right.$ $\mathrm{MHz}) \delta: 7.30 \sim 7.07$ (m, 8H), 6.57 (brs, $2 \mathrm{H}), 4.77 \sim 4.72$ $(\mathrm{m}, 2 \mathrm{H}), 3.89(\mathrm{~s}, 2 \mathrm{H}), 2.05 \sim 1.26(\mathrm{~m}, 20 \mathrm{H})$.

二苯甲烷二氨基甲酸二庚酯(3I): 白色固体. m.p. $110{ }^{\circ} \mathrm{C}$ (CAS No. 299202-68-9); ${ }^{1} \mathrm{H}$ NMR $\left(\mathrm{CDCl}_{3}, 400\right.$ MHz) $\delta: 7.30 \sim 7.09(\mathrm{~m}, 8 \mathrm{H}), 6.54(\mathrm{brs}, 2 \mathrm{H}), 4.15(\mathrm{t}, J=$ $6.8 \mathrm{~Hz}, 4 \mathrm{H}), 3.89(\mathrm{~s}, 2 \mathrm{H}), 1.70 \sim 1.63(\mathrm{~m}, 4 \mathrm{H}), 1.39 \sim 1.26$ $(\mathrm{m}, 16 \mathrm{H}), 0.89(\mathrm{t}, J=6.8 \mathrm{~Hz}, 6 \mathrm{H})$.

二苯甲烷二氨基甲酸二辛酯 $(\mathbf{3 m})$ : 白色固体. m.p. $120{ }^{\circ} \mathrm{C}$ (lit. $\left.{ }^{[24]} 117 \sim 119{ }^{\circ} \mathrm{C}\right) ;{ }^{1} \mathrm{H}$ NMR $\left(\mathrm{CDCl}_{3}, 400\right.$ $\mathrm{MHz}) \delta: 7.30 \sim 7.07(\mathrm{~m}, 8 \mathrm{H}), 6.53$ (brs, $2 \mathrm{H}), 4.15(\mathrm{t}, J=$ $6.6 \mathrm{~Hz}, 4 \mathrm{H}), 3.89(\mathrm{~s}, 2 \mathrm{H}), 1.70 \sim 1.58(\mathrm{~m}, 4 \mathrm{H}), 1.37 \sim 1.23$ (m, 20H), 0.89 (t, $J=6.4 \mathrm{~Hz}, 6 \mathrm{H})$.

辅助材料(Supporting Information) 化合物原始核磁谱 图. 这些材料可以免费从本刊网站 (http://sioc-journal. $\mathrm{cn} /$ )上下载.

\section{References}

[1] (a) Jia C. Q.; Yang D. Y.; Che C. L.; Ma Y. Q.; Rui C. H.; Yan X. J.; Tan Z. H. Chem. J. Chin. Univ. 2016, 37, 892 (in Chinese). (贾长青, 杨冬燕, 车传亮, 马永强, 苪昌辉, 问晓静, 覃兆海, 高等学校化学学报, 2016, 37, 892.)

(b) Wu, X. P.; Wang, L.; Xie, Z. H.; Yan, C.; Chen, G. N. Chem. Res. Chin. Univ. 2007, 23, 109.

(c) Gupte, S. P.; Shivarkar, A. B.; Chaudhari, R. V. Chem. Commun. 2001, 2620.

(d) Kodaka, R.; Sugano, T.; Katagi, T.; Takimoto, Y. J. Agric. Food Chem. 2003, 51, 7730 .

(e) Srivastava, R.; Manju, M. D.; Srinivas, D.; Ratnasamy, P. Catal. Lett. 2004, 97, 41.

[2] (a) Tafesh, A. M.; Weiguny, J. Chem. Rev. 1996, 96, 2035.

(b) Paul, P. Coord. Chem. Rev. 2000, 203, 269.

(c) Baba, T.; Kobayashi, A.; Kawanami, Y.; Inazu, K.; Ishikawa, A.; Echizenn, T.; Murai, K.; Aso, S.; Inomata, M. Green Chem. 2005, 7,159 .

(d) Shi, F.; Deng, Y. Q.; Sima, T. L.; Yang, H. Z. J. Catal. 2001, 203, 525 .

[3] (a) White, J. D.; Blakemore, P. R.; Milicevic, S. Org. Lett. 2002, 4, 1803.

(b) Feroci, M.; Casadei, M. A.; Orsini, M.; Palombi, L.; Ines, A. J. Org. Chem. 2003, 68, 1548.

(c) Deleon, R. G.; Kobayashi, A.; Yamauchi, T.; Ooishi, J.; Baba, T.; Sasaki, M.; Hiarata, F. Appl. Catal., A 2002, 225, 43.

[4] (a) Crosby, D. G.; Niemann, C. J. Am. Chem. Soc. 1954, 76, 4458. (b) Zhang, A.; Kuwahara, Y.; Hotta, Y.; Tsuda, A. Asian J. Org. Chem. 2013, 2, 572.

[5] (a) Uhlig, N.; Li, C. J. Chem.-Eur. J. 2014, 20, 12066.

(b) Seth, K.; Nautiyal, M.; Purohit, P.; Parikh, N.; Chakraborti, A. K. Chem. Commun. 2015, 51, 191.

(c) Izawa, Y.; Ishiguro, K.; Tomioka, H. Bull. Chem. Soc. Jpn. 
1983, 56, 951 .

(d) Padiya, K. J.; Gavade, S.; Kardile, B.; Tiwari, M.; Bajare, S.; Mane, M.; Gaware, V.; Varghese, S.; Harel, D.; Kurhade, S. Org. Lett., 2012, 14, 2814.

(e) Kang, W. K.; Wang, G. Y.; Yao, J. Chem. Res. Chin. Univ. 2006, 22,669 .

[6] Ragaini, F.; Gasperini, M.; Cenini, S. Adv. Synth. Catal. 2004, 346, 63.

[7] Yang, Y.; Lu, S. W. Chin. J. Catal. 1999, 20, 224 (in Chinese). (杨瑛, 陆世维, 催化学报, 1999, 20, 224.)

[8] (a) Shi, F.; Sima, T. L.; Deng, Y. Q. Chem. J. Chin. Univ. 2000, 21, 1566 (in Chinese).

(石峰, 司马天龙, 邓友全, 高等学校化学学报, 2000, 21, 1566.) (b) Mei, F. M.; Chen, L. J.; Li, G. X. Appl. Organomet. Chem. 2010, 24, 86.

(c) Shi, F.; Deng, Y. Q. Chem. Commun. 2001, 443.

[9] Stock, C.; Brueckner, R. Adv. Synth. Catal. 2012, 354, 2309.

[10] (a) Pei, Y. X.; Li, H. Q.; Liu, H. T.; Zhang, Q. H.; Zhang, Y. Chem. Res. Chin. Univ. 2010, 26, 550.

(b) Kim, S. D.; Lee, K. H. J. Mol. Catal. 1993, 78, 237.

[11] (a) Zhang, X. P.; Miao, J. H.; Sun, Y. B. Chin. J. Org. Chem. 2009, 29, 1555 (in Chinese).

(张晓鹏, 苗江欢, 孙玉标, 有机化学, 2009, 29, 1555.)

(b) Sonoda, N. Pure Appl. Chem. 1993, 65, 699.

[12] Zhang, X. P.; Li, D. S.; Ma, X. J.; Wang, Y.; Zhang, G. S. Synthesis
2013, 45, 1357.

[13] Kang, L. J.; Zhao, X. Q.; An, H. L.; Wan, Y. J. Acta Pet. Sin. 2013, 29, 249 (in Chinese).

(康丽娟, 赵新强, 安华良, 王延吉, 石油学报, 2013, 29, 249.)

[14] Kianmehr, E.; Baghersad, M. H. Adv. Synth. Catal. 2011, 353, 2599.

[15] George, D. K.; Moore, D. H.; Brian, W. P.; Garman, J. A. J. Agric. Food Chem. 1954, 2, 356.

[16] Whitmore, F. C.; Popkin, A. H.; Whitaker, J. S.; Mattil, K. F.; Zech, J. D. J. Am. Chem. Soc. 1938, 60, 2462.

[17] Schicktanz, S. T.; Etienne, A. D.; Steele, W. I. Ind. Eng. Chem. Anal. Ed. 1939, 11, 420.

[18] Bayer, F. GB 775723, 1957 [Chem. Abstr. 1957, 51, 90827].

[19] Brockway, C. E. US 2806051, 1957 [Chem. Abstr. 1957, 52, 15931].

[20] Murphy, W. S.; Raman, K. P. J. Chem. Soc., Perkin Trans. 1 1981, 447.

[21] Matthews, K. H.; Mclennaghan, A.; Pethrick, R. A. Brit. Polym. J. 1987, 19, 165.

[22] Tereshatov, V. V.; Senichev, V. Y. J. Appl. Polym. Sci. 2015, 41481.

[23] Chapman,T. M. J. Appl. Polym. Sci. Part A: Polym. Chem. 1989, 27, 1993.

[24] Lu, Q. W.; Hoye, T. R.; Macosko, C. W. Thermoplastic Polyurethane Blends 2002, 2310.

(Li, L.; Fan, Y.) 